\title{
On the sum of distance Laplacian eigenvalues of graphs
}

\author{
Shariefuddin Pirzada and Saleem Khan
}

\begin{abstract}
Let $G$ be a connected graph with $n$ vertices, $m$ edges and having diameter d. The distance Laplacian matrix $D^{L}$ is defined as $D^{L}=\operatorname{Diag}(T r)-D$, where $\operatorname{Diag}(T r)$ is the diagonal matrix of vertex transmissions and $D$ is the distance matrix of $G$. The distance Laplacian eigenvalues of $G$ are the eigenvalues of $D^{L}$ and are denoted by $\delta_{1}, \delta_{1}, \ldots, \delta_{n}$. In this paper, we obtain (a) the upper bounds for the sum of $k$ largest and (b) the lower bounds for the sum of $k$ smallest non-zero, distance Laplacian eigenvalues of $G$ in terms of order $n$, diameter $d$ and Wiener index $W$ of $G$. We characterize the extremal cases of these bounds. Also, we obtain the bounds for the sum of the powers of the distance Laplacian eigenvalues of $G$. Finally, we obtain a sharp lower bound for the sum of the $\beta$ th powers of the distance Laplacian eigenvalues, where $\beta \neq 0,1$.
\end{abstract}

Keywords. Distance matrix, distance Laplacian matrix, distance Laplacian eigenvalues, diameter, Wiener index

\section{Introduction}

A graph $G=(V, E)$ consists of the vertex set $V(G)=\left\{v_{1}, v_{2}, \ldots, v_{n}\right\}$ and the edge set $E(G)$. We assume all the graphs under consideration are simple and connected. Further, $|V(G)|=n$ is the order and $|E(G)|=m$ is the size of $G$. The degree of $v$, denoted by $d_{G}(v)$ (we simply write $d_{v}$ ) is the number of edges incident on the vertex $v$. As usual, $K_{n}$ is a complete graph with $n$ vertices and $K_{1, n-1}$ is a star graph with $n$ vertices. Also, $K_{a, b}$ is a complete bipartite graph with two partite sets $V_{1}$ and $V_{2}$ of cardinalities $a$ and $b$, respectively, such that each vertex of $V_{1}$ is adjacent to every vertex of $V_{2}$. For other standard definitions, we refer [12].

The adjacency matrix $A=\left(a_{i j}\right)$ of $G$ is an $n \times n$ matrix whose $(i, j)$-entry is equal to 1 , if $v_{i}$ is adjacent to $v_{j}$ and equal to 0 , otherwise. Let $\operatorname{Deg}(G)=\operatorname{diag}\left(d_{1}, d_{2}, \ldots, d_{n}\right)$ be the diagonal matrix of vertex degrees $d_{i}=d_{v_{i}}, i=1,2, \ldots, n$ of $G$. The positive semi-definite matrix $L(G)=\operatorname{Deg}(G)-A(G)$ is the Laplacian matrix of $G$. The eigenvalues of $L(G)$ are called the Laplacian eigenvalues of $G$. Let $S_{k}(G)$ be the sum of the $k$ largest Laplacian eigenvalues of $G$. Several researchers have been investigating the parameter $S_{k}(G)$ because of its importance in dealing with many problems in the theory, for instance, Brouwer's conjecture, Laplacian energy. More recent work on $S_{k}(G)$ can be seen in $[5,6,7,8,13]$.

Received date: February 13, 2021; Published online: November 7, 2021. 2010 Mathematics Subject Classification. 05C50, 05C12, $15 \mathrm{~A} 18$.

Corresponding author: Shariefuddin Pirzada. 
In $G$, the distance between two vertices $u, v \in V(G)$, denoted by $d_{u v}$, is defined as the length of a shortest path between $u$ and $v$. The diameter of $G$ is the maximum distance between any two vertices of $G$. The distance matrix of $G$ is denoted by $D(G)$ and is defined as $D(G)=\left(d_{u v}\right)_{u, v \in V(G)}$. The vertex transmission $\operatorname{Tr}_{G}(v)$ of a vertex $v$ is defined as the sum of the distances from $v$ to all other vertices in $G$, that is, $\operatorname{Tr}_{G}(v)=\sum_{u \in V(G)} d_{u v}$. A graph $G$ is said to be $k$-transmission regular if $\operatorname{Tr}_{G}(v)=k$, for each $v \in V(G)$. The Wiener index (also called transmission) of a graph $G$, denoted by $W(G)$, is the sum of distances between all unordered pairs of vertices in $G$. Clearly, $W(G)=\frac{1}{2} \sum_{v \in V(G)} \operatorname{Tr}_{G}(v)$. For any vertex $v_{i} \in V(G)$, the vertex transmission $\operatorname{Tr}_{G}\left(v_{i}\right)$ is also called the transmission degree of $v_{i}$.

Let $\operatorname{Tr}(G)=\operatorname{diag}\left(\operatorname{Tr}_{1}, \operatorname{Tr}_{2}, \ldots, T r_{n}\right)$ be the diagonal matrix of vertex transmissions of $G$. Aouchiche and Hansen [1] introduced the Laplacian for the distance matrix of a connected graph. The matrix $D^{L}(G)=\operatorname{Tr}(G)-D(G)$ (or simply $D^{L}$ ) is called the distance Laplacian matrix of $G$. The eigenvalues of $D^{L}$ are called the distance Laplacian eigenvalues of $G$. Since $D^{L}(G)$ is a real symmetric positive semi-definite matrix, we denote its eigenvalues by $\delta_{i}$ 's and order them as $0=\delta_{n} \leq \delta_{n-1} \leq \cdots \leq \delta_{1}$. The largest distance Laplacian eigenvalue $\delta_{1}$ is called the distance Laplacian spectral radius of $G$. More work on distance Laplacian eigenvalues can be found in $[2,9,11]$.

Motivated by the parameter $S_{k}(G)$ of the Laplacian matrix, we introduce the following. For $1 \leq k \leq n-1$, let $U_{k}$ denote the sum of the $k$ largest distance Laplacian eigenvalues and $L_{k}$ denote the sum of $k$ smallest positive distance Laplacian eigenvalues of the graph $G$, that is,

$$
U_{k}=\sum_{i=1}^{k} \delta_{i} \quad \text { and } \quad L_{k}=\sum_{i=1}^{k} \delta_{n-i} .
$$

In Section 2, we obtain the upper bounds for $U_{k}$ in terms of order $n$, diameter $d$ and Wiener index $W$ of $G$. Also, we find the lower bounds for $L_{k}$ in terms of the same parameters. In particular, we obtain the bounds for $U_{k}$ and $L_{k}$ when $G$ is a bipartite graph. We characterize the extremal cases of these bounds. We derive the bounds for the sum of the powers of the distance Laplacian eigenvalues of $G$. Also, we obtain a sharp lower bound for the sum of the $\beta$ th powers of the distance Laplacian eigenvalues, where $\beta \neq 0,1$.

\section{On the sum of the distance Laplacian eigenvalues of graphs}

We begin with the following observation due to Caen [3].

Lemma 2.1. [3] Let $[n]=\{1,2, \ldots, n\}$ be the canonical $n$-element set and let $[n]^{(2)}$ denote the set of 2-element subsets of $[n]$, that is, the edge set of $K_{n}$. To each entry $\{i, j\}=i j$ in $[n]^{(2)}$, associate a real variable $x_{i j}$, then for $n \geq 2$, and for all $x_{i j}^{\prime} s$, we have

$$
\left(\sum_{i j} x_{i j}\right)^{2}+\left(\begin{array}{c}
n-1 \\
2
\end{array}\right) \sum_{i j} x_{i j}^{2}-\frac{n-1}{2} \sum_{i}\left(\sum_{j \neq i} x_{i j}\right)^{2} \geq 0 .
$$

The following result gives the upper bound for the sum of the squares of the vertex transmissions in terms of the Wiener index $W$, the diameter $d$ and the order $n$ of the graph $G$. 
Lemma 2.2. Let $G$ be a connected graph with $n$ vertices having diameter d. Then

$$
\sum_{i} \operatorname{Tr}^{2}(i) \leq \frac{2 W^{2}}{n-1}+\frac{n(n-1)(n-2) d^{2}}{2}
$$

with equality if and only if $G \cong K_{n}$.

Proof. Substituting $d_{i j}$ for $x_{i j}$ in Lemma 2.1 and noting that each $d_{i j} \leq d$, we have

$$
\begin{aligned}
& \left(\sum_{i j} d_{i j}\right)^{2}+\left(\begin{array}{c}
n-1 \\
2
\end{array}\right) \sum_{i j} d_{i j}^{2}-\frac{n-1}{2} \sum_{i}\left(\sum_{j \neq i} d_{i j}\right)^{2} \geq 0 \\
& \text { or } \quad W^{2}+\left(\begin{array}{c}
n-1 \\
2
\end{array}\right) \sum_{i j} d_{i j}^{2}-\frac{n-1}{2} \sum_{i} \operatorname{Tr}^{2}(i) \geq 0 .
\end{aligned}
$$

Then $\sum_{i} \operatorname{Tr}^{2}(i) \leq \frac{2 W^{2}}{n-1}+\frac{2}{n-1} \frac{(n-1)(n-2)}{2}\left(\frac{n(n-1)}{2} d^{2}\right)$,

$$
\text { or } \sum_{i} \operatorname{Tr}^{2}(i) \leq \frac{2 W^{2}}{n-1}+\frac{n(n-1)(n-2) d^{2}}{2},
$$

proving the inequality.

Assume that the equality holds in the above inequalities. Then clearly each $d_{i j}=d$. Since $G$ is connected, there is at least one $d_{i j}=1$ and thus $d=1$ which clearly shows that $G \cong K_{n}$.

Conversely, if $G \cong K_{n}$, then it is easy to see that $d=1, W=\frac{n(n-1)}{2}$ and $\sum_{i} \operatorname{Tr}^{2}(i)=$ $n(n-1)^{2}$. Substituting these values in the above inequalities, we observe that the equality holds.

Now, we obtain an upper bound for $U_{k}$ in terms of the Wiener index, the diameter and the order of the graph $G$. The proof follows by using similar techniques as in Zhou [14].

Theorem 2.1. Let $G$ be a connected graph with $n$ vertices having diameter $d$. For $1 \leq k \leq n-2$, we have

$$
U_{k} \leq \frac{2 W k}{n-1}+\frac{\sqrt{k(n-k-1)(d n(n-1)-2 W)(d n(n-1)+2 W)}}{\sqrt{2}(n-1)}
$$

with equality if and only if $G \cong K_{n}$. Equality always holds for $k=n-1$.

Proof. It is easy to see that

$$
\sum_{i=1}^{n-1} \delta_{i}=\sum_{i \in V} \operatorname{Tr}(i)=2 W \text { and } \sum_{i=1}^{n-1} \delta_{i}^{2}=\sum_{i} \operatorname{Tr}^{2}(i)+2 \sum_{1 \leq i<j \leq n} d_{i j}^{2} .
$$

For $1 \leq k \leq n-2$, using Cauchy-Schwarz inequality, we have

$$
\begin{aligned}
& \left(2 W-U_{k}\right)^{2} \\
& =\left(\delta_{k+1}+\cdots+\delta_{n-1}\right)^{2} \leq(n-k-1)\left(\delta_{k+1}^{2}+\cdots+\delta_{n-1}^{2}\right) \\
& =(n-k-1)\left(\sum_{i} \operatorname{Tr}^{2}(i)+2 \sum_{1 \leq i<j \leq n} d_{i j}^{2}-\left(\delta_{1}^{2}+\cdots+\delta_{k}^{2}\right)\right) \\
& \leq(n-k-1)\left(\sum_{i} \operatorname{Tr}^{2}(i)+2 \sum_{1 \leq i<j \leq n} d_{i j}^{2}-\frac{U_{k}^{2}}{k}\right),
\end{aligned}
$$


which implies that

$$
U_{k}^{2}-\frac{4 k W U_{k}}{n-1}+\frac{4 k W^{2}}{n-1}-\frac{k(n-k-1)}{n-1}\left(\sum_{i} \operatorname{Tr}^{2}(i)+2 \sum_{1 \leq i<j \leq n} d_{i j}^{2}\right) \leq 0 .
$$

Thus,

$$
U_{k} \leq \frac{2 W k+\sqrt{k(n-k-1)\left[(n-1)\left(\sum_{i} \operatorname{Tr}^{2}(i)+2 \sum_{1 \leq i<j \leq n} d_{i j}^{2}\right)-4 W^{2}\right]}}{n-1} .
$$

Using Lemma 2.2, we get

$$
U_{k} \leq \frac{2 W k+\sqrt{k(n-k-1)\left[(n-1)\left(\frac{2 W^{2}}{n-1}+\frac{n(n-1)(n-2) d^{2}}{2}+d^{2} n(n-1)\right)-4 W^{2}\right]}}{n-1} .
$$

On further simplifications, we have

$$
U_{k} \leq \frac{2 W k+\sqrt{\frac{k(n-k-1)\left(d^{2} n^{2}(n-1)^{2}-4 W^{2}\right)}{2}}}{n-1},
$$

or,

$$
U_{k} \leq \frac{2 W k}{n-1}+\frac{\sqrt{k(n-k-1)(d n(n-1)-2 W)(d n(n-1)+2 W)}}{\sqrt{2}(n-1)},
$$

proving the inequalities.

Assume that the equality hold in above inequalities. Then all the above inequalities have to be equalities and after using Cauchy-Schwarz theorem and Lemma 2.2, we observe that $G \cong K_{n}$.

Conversely, let $G \cong K_{n}$. Taking the characteristic polynomial of $K_{n}$ into consideration, it is quite easy to check that $U_{k}=n k, W=\frac{n(n-1)}{2}$ and $d=1$. Using these values in the main inequality, we observe that the equality holds.

Using the fact that trace of a matrix is equal to sum of its eigenvalues and noting that $2 W=U_{n-1}$, we see that equality always holds in main inequality when $k=n-1$.

From Theorem 2.1, we obtain the following upper bound for the spectral radius $\delta_{1}$ of the distance Laplacian matrix of a graph.

Theorem 2.2. Let $G$ be a connected graph on $n$ vertices having diameter $d$. Then

$$
\delta_{1} \leq \frac{2 W}{n-1}+\frac{\sqrt{(n-2)(d n(n-1)-2 W)(d n(n-1)+2 W)}}{\sqrt{2}(n-1)}
$$

with equality if and only if $G \cong K_{n}$.

Using arguments same as in Theorem 2.1, we have the following lower bound for $L_{k}$.

Theorem 2.3. Let $G$ be a connected graph with $n$ vertices having diameter $d$. Then, for $1 \leq$ $k \leq n-2$, we have

$$
L_{k} \geq \frac{2 W k}{n-1}-\frac{\sqrt{k(n-k-1)(d n(n-1)-2 W)(d n(n-1)+2 W)}}{\sqrt{2}(n-1)}
$$

with equality if and only if $G \cong K_{n}$. Equality always holds for $k=n-1$. 
As a consequence of Theorem 2.3, we get the following upper bound for the smallest non-zero distance Laplacian eigenvalue $\delta_{n-1}$ of $G$.

$$
\delta_{n-1} \geq \frac{2 W}{n-1}-\frac{\sqrt{(n-2)(d n(n-1)-2 W)(d n(n-1)+2 W)}}{\sqrt{2}(n-1)}
$$

with equality if and only if $G \cong K_{n}$.

Now, we have the following observation about the sum of the squares of the distances in a graph.

Lemma 2.3. Let $G$ be a connected graph with $n \geq 2$ vertices and $m$ edges having diameter $d$. Then

$$
\sum_{1 \leq i<j \leq n} d_{i j}^{2} \leq \frac{2 m\left(1-d^{2}\right)+d^{2} n(n-1)}{2}
$$

with equality if and only if $d \leq 2$.

Proof. Since $G$ contains $m$ edges, therefore there are exactly $m$ distances equal to 1 and the remaining distances (if there are any) are greater or equal to 2 . As each $d_{i j} \leq d$, we have

$$
\begin{aligned}
\sum_{1 \leq i<j \leq n} d_{i j}^{2} & \leq m+d^{2}\left(\frac{n(n-1)}{2}-m\right) \\
& =\frac{2 m\left(1-d^{2}\right)+d^{2} n(n-1)}{2},
\end{aligned}
$$

proving the inequality.

Assume that the equality hold in above inequalities. If there are no non-adjacent pair of vertices, then clearly $d=1$, so that $G$ is a complete graph. If there are some non-adjacent pair of vertices, then from the above proof we observe that the distance between every non-adjacent pair of vertices is same and equals to $d$. Since $G$ is connected, at least one such distance equals to 2 , so that $d=2$.

Conversely, if $d=1$, then $G$ is a complete graph and it is easy to check that the equality holds in this case. Similarly, we can easily see that the equality holds when $d=2$. This proves the result.

From Lemma 2.3, we obtain the following corollary for the class of connected bipartite graphs.

Corollary 2.4. Let $G$ be a connected bipartite graph with $n \geq 3$ vertices, $m$ edges and having diameter $d$. Then

$$
\sum_{1 \leq i<j \leq n} d_{i j}^{2} \leq \frac{2 m\left(1-d^{2}\right)+d^{2} n(n-1)}{2}
$$

with equality if and only if $G$ is a complete bipartite graph.

We require the following lemma due to Zhou [14].

Lemma 2.4. [14] Let $G$ be a connected bipartite graph on $n$ vertices and $m$ edges and let $d_{v}$ be the degree of any vertex $v$ of $G$. Then

$$
\sum_{v \in V(G)} d_{v}^{2} \leq m n
$$

with equality if and only if $G$ is a complete bipartite graph. 
We have the following observation for the sum of the squares of the vertex transmissions of a bipartite graph.

Lemma 2.5. Let $G$ be a connected bipartite graph having $n \geq 3$ vertices, $m$ edges and diameter $d$ with bi-partition of vertex set as $\left\{V_{1}, V_{2}\right\}$ with $\left|V_{1}\right|=a,\left|V_{2}\right|=b, a \geq b \geq 1$ and $a+b=n$. Then

$$
\sum_{i} \operatorname{Tr}^{2}(i) \leq m n(1-d)^{2}+n d^{2}(n-1)^{2}+4 m d(1-d)(n-1)
$$

with equality if and only if $G$ is a complete bipartite graph.

Proof. Without loss of generality, let $i \in V_{1}$. Since $G$ is bipartite we have the following inequality

$$
\operatorname{Tr}(i) \leq d_{i}+d\left(b-d_{i}\right)+d(a-1)
$$

or,

$$
\operatorname{Tr}(i) \leq d_{i}(1-d)+d(n-1)
$$

so that

$$
\operatorname{Tr}^{2}(i) \leq d_{i}^{2}(1-d)^{2}+(d(n-1))^{2}+2 d d_{i}(1-d)(n-1) .
$$

Taking the summation over all vertices in $V$, we have

$$
\sum_{i} \operatorname{Tr}^{2}(i) \leq \sum_{i} d_{i}^{2}(1-d)^{2}+\sum_{i}(d(n-1))^{2}+\sum_{i} 2 d d_{i}(1-d)(n-1) .
$$

Using Lemma 2.4 and noting that $\sum_{i} d_{i}=2 m$, from the above inequality, we have

$$
\sum_{i} \operatorname{Tr}^{2}(i) \leq m n(1-d)^{2}+n d^{2}(n-1)^{2}+4 m d(1-d)(n-1)
$$

Assume that the equality hold in above inequality. Then we observe that the distance between every non-adjacent pair of vertices is the same and equals to 2 , which is possible only if $G$ is complete bipartite.

Conversely, it is easy to check that the equality holds for complete bipartite graphs.

We note the following observation.

Lemma 2.6. [1] The distance Laplacian characteristic polynomial of a complete bipartite graph $K_{a, b}$ is $P_{L}^{K_{a, b}}(x)=x(x-n)(x-(2 a+b))^{a-1}(x-(2 b+a))^{b-1}$.

Now, we obtain an upper bound for $U_{k}$ when $G$ is a connected bipartite graph.

Theorem 2.5. Let $G$ be a connected bipartite graph with $n \geq 3$ vertices, $m$ edges and having diameter $d$. Then, for $1 \leq k \leq n-2$, we have

$$
U_{k} \leq \frac{2 W k+\sqrt{k(n-k-1)\left[(n-1)\left(m(1-d)(n+3 d n-2 d+2)+d^{2} n^{2}(n-1)\right)-4 W^{2}\right]}}{n-1}
$$

with equality if and only if $k=n-2$ and $G \cong K_{1, n-1}$ or $G \cong K_{\frac{n}{2}, \frac{n}{2}}$. Equality always holds for $k=n-1$. 
Proof. Let $1 \leq k \leq n-2$. From the proof of Theorem 2.1 and using Corollary 2.4 and Lemma 2.5 , we get the required inequality.

Let the equality hold in above inequality. Then we observe that

$$
\delta_{1}=\delta_{2}=\cdots=\delta_{k} \text { and } \delta_{k+1}=\delta_{k+2}=\cdots=\delta_{n-1}
$$

and $G$ is a complete bipartite graph. Taking Lemma 2.6 into consideration, we have $k=n-2$ and $G \cong K_{1, n-1}$ or $G \cong K_{\frac{n}{2}, \frac{n}{2}}$.

Conversely, if $k=n-2$ and $G \cong k_{1, n-1}$ or $G \cong k_{\frac{n}{2}, \frac{n}{2}}$, then we can easily check that equality follows in both cases.

Using same argument as in Theorem 2.1, we see that equality holds when $k=n-1$.

Some results for $\delta_{1}$ in bipartite graphs can be seen in [10]. Using Theorem 2.5, we get the following strict upper bound for distance Laplacian spectral radius for the class of connected bipartite graphs with $n \geq 4$.

Theorem 2.6. Let $G$ be a connected bipartite graph with $n \geq 4$ vertices, $m$ edges and having diameter $d$. Then

$$
\delta_{1}<\frac{2 W+\sqrt{(n-2)\left[(n-1)\left(m(1-d)(n+3 d n-2 d+2)+d^{2} n^{2}(n-1)\right)-4 W^{2}\right]}}{n-1} .
$$

With the help of Theorem 2.5, we can easily obtain the following upper bound for $L_{k}$.

Theorem 2.7. Let $G$ be a connected bipartite graph with $n \geq 3$ vertices, $m$ edges and having diameter $d$. Then, for $1 \leq k \leq n-2$, we have

$$
L_{k} \geq \frac{2 W k-\sqrt{k(n-k-1)\left[(n-1)\left(m(1-d)(n+3 d n-2 d+2)+d^{2} n^{2}(n-1)\right)-4 W^{2}\right]}}{n-1}
$$

with equality if and only if $k=n-2$ and $G \cong K_{1, n-1}$ or $G \cong K_{\frac{n}{2}, \frac{n}{2}}$. Equality always holds for $k=n-1$.

The following lemma will be used in the sequel.

Lemma 2.7. [1] Let $G$ be a connected graph on $n$ vertices and $m \geq n$ edges. Let $G^{*}$ be the connected graph obtained from $G$ by the deletion of an edge. Let $\delta_{1} \geq \delta_{2} \geq \cdots \geq \delta_{n-1} \geq \delta_{n}=0$ and $\delta_{1}^{*} \geq \delta_{2}^{*} \geq \cdots \geq \delta_{n-1}^{*} \geq \delta_{n}^{*}=0$ denote the distance Laplacian spectra of $G$ and $G^{*}$, respectively. Then, $\delta_{i}^{*} \geq \delta_{i}$, for all $i=1,2, \ldots, n$.

Now, let $\beta$ be a real number such that $\beta \neq 0,1$ and let $S_{\beta}(G)=\sum_{i=1}^{n-1} \delta_{i}^{\beta}$, that is, the sum of the $\beta$ th powers of distance Laplacian eigenvalues of $G$.

Lemma 2.8. Let $G$ be a connected graph with $n$ vertices and $m \geq n$ edges. Let $G^{*}$ be the connected graph obtained from $G$ by the deletion of an edge. Let $\delta_{1} \geq \delta_{2} \geq \cdots \geq \delta_{n-1} \geq \delta_{n}=0$ and $\delta_{1}^{*} \geq \delta_{2}^{*} \geq \cdots \geq \delta_{n-1}^{*} \geq \delta_{n}^{*}=0$ be the distance Laplacian eigenvalues of $G$ and $G^{*}$, respectively. Then

(i) $S_{\beta}\left(G^{*}\right)>S_{\beta}(G)$ for $\beta>0$ and $S_{\beta}\left(G^{*}\right)<S_{\beta}(G)$ for $\beta<0$.

(ii) For any connected graph $G$ with $n$ vertices $S_{\beta}(G) \geq(n-1) n^{\beta}$ if $\beta>0$, and $S_{\beta}(G) \leq(n-1) n^{\beta}$ if $\beta<0$, with either of the equality if and only if $G \cong K_{n}$. 
Proof. Using Lemma 2.7, we have $\sum_{i=1}^{n-1} \delta_{i}^{*}-\sum_{i=1}^{n-1} \delta_{i} \geq 2$. Hence (i) follows directly from Lemma 2.7. We know that the eigenvalues of $K_{n}$ are $n, n, \ldots, n, 0$, so that (ii) follows from (i).

The following lemma will be used in the proof of Theorem 2.8.

Lemma 2.9. [4] Let $G$ be a connected graph such that $D^{L}$ has an eigenvalue with multiplicity $n-2$. Let $\delta_{1} \geq \delta_{2} \geq \cdots \geq \delta_{n-1} \geq \delta_{n}=0$ be the eigenvalues of $D^{L}$. Then exactly one of the following condition holds.

(i) $m\left(\delta_{1}\right)=n-2$ and $G \cong K_{1, n-1}$ or $G \cong K_{p, p}$.

(ii) $m\left(\delta_{n-1}\right)=n-2$ and $G \cong K_{n-2} \vee \overline{K_{2}}$.

Now, we have the following result.

Theorem 2.8. Let $\beta$ be a real number with $\beta \neq 0,1$ and let $G$ be a connected graph with $n \geq 3$ vertices. Let $R=\prod_{i=1}^{n-1} \delta_{i}$. Then

$$
S_{\beta}(G) \geq \delta_{1}^{\beta}+(n-2)\left(\frac{R}{\delta_{1}}\right)^{\frac{\beta}{n-2}}
$$

with equality if and only if $G \cong K_{n}$ or $G \cong K_{n-2} \vee \overline{K_{2}}$.

Proof. By the arithmetic-geometric mean inequality, we have

$$
\begin{aligned}
S_{\beta}(G) & =\delta_{1}^{\beta}+\sum_{i=2}^{n-1} \delta_{i}^{\beta} \geq \delta_{1}^{\beta}+(n-2)\left(\prod_{i=2}^{n-1} \delta_{i}^{\beta}\right)^{\frac{1}{n-2}} \\
& =\delta_{1}^{\beta}+(n-2)\left(\frac{\prod_{i=1}^{n-1} \delta_{i}}{\delta_{1}}\right)^{\frac{\beta}{n-2}}=\delta_{1}^{\beta}+(n-2)\left(\frac{R}{\delta_{1}}\right)^{\frac{\beta}{n-2}}
\end{aligned}
$$

with equality if and only if $\delta_{2}=\delta_{3}=\cdots=\delta_{n-1}$.

Now, if $\delta_{1}=\delta_{2}$, then $\delta_{1}=\delta_{2}=\delta_{3}=\cdots=\delta_{n-1}$, so that $G \cong K_{n}$. If $\delta_{1} \neq \delta_{2}$, by Lemma 2.9, we have $G \cong K_{n-2} \vee \overline{K_{2}}$.

Conclusions. The parameters $U_{k}$ and $L_{k}$ introduced in this paper will be of great importance in the investigation of the distribution of distance Laplacian eigenvalues of a graph. In particular, it will throw more light in the study of the distance Laplacian spectral radius and distance Laplacian energy of a graph. Although the bounds for $U_{k}$ and $L_{k}$ are obtained in terms of the order, Wiener index and diameter of the graph, there is enough scope to obtain more bounds for $U_{k}$ and $L_{k}$ in terms of several other parameters, which will lead to interesting discussion on the problem.

\section{Acknowledgments}

We are highly thankful to the anonymous referees for their valuable suggestions. The research of S. Pirzada is supported by the SERB-DST research project number CRG/2020/000109. The research of Saleem Khan is supported by MANUU. 


\section{References}

[1] M. Aouchiche and P. Hansen, Two Laplacians for the distance matrix of a graph, Linear Algebra Appl., 439 (2013), 21-33.

[2] M. Aouchiche and P. Hansen, Some properties of the distance Laplacian eigenvalues of a graph, Czechoslovak Mathematical Journal, 64(139) (2014), 751-761.

[3] D. D. Caen An upper bound on the sum of squares of degrees in a graph, Discrete Math., 185 (1998), 245-248.

[4] R. Fernandes, M. A. A. de Freitas, C.M. da Silva Jr and R. R. Del-Vecchio, Multiplicities of distance Laplacian eigenvalues and forbidden subgraphs, Linear Algebra Appl., 541 (2018), $81-93$.

[5] H. A. Ganie, S. Pirzada and Vilmar Trevisan, On the sum of $k$ largest Laplacian eigenvalues of a graph and clique number, Mediterranean J. Math., 18 (2021), Artcle No. 15.

[6] H. A. Ganie, S. Pirzada, Bilal A. Rather and Rezwan Ul Shaban, On Laplacian eigenvalues of graphs and Brouwer's conjecture, J. Ramanujan Math. Soc., 36(1) (2021), 13-21.

[7] H. A. Ganie, S. Pirzada, Bilal A. Rather and Vilmar Trevisan, Further developments on Brouwer's conjecture for the sum of Laplacian eigenvalues of graphs, Linear Algebra Appl., 588 (2020), 1-18.

[8] H. A. Ganie, S. Pirzada, Rezwan Ul Shaban and X. Li, Upper bounds for the sum of Laplacian eigenvalues of a graph and Brouwer's conjecture, Discrete Math. Algorithms Appl., 11(2) (2019), 195008 (15 pages).

[9] M. Nath and S. Paul, On the distance Laplacian spectra of graphs, Linear Algebra Appl., 460 (2014), 97-110.

[10] A. Niu, D. Fan and G. Wang, On the distance Laplacian spectral radius of bipartite graphs, Discrete Appl. Math., 186 (2015), 207-213.

[11] S. Pirzada and Saleem Khan, On distance Laplacian spectral radius and chromatic number of graphs, Linear Algebra Appl., 625 (2021), 44-54.

[12] S. Pirzada, An Introduction to Graph Theory, $1^{\text {st }}$ edition, Universities Press, Orient Blackswan, Hyderabad, 2012.

[13] S. Pirzada and H. A. Ganie, On the Laplacian eigenvalues of a graph and Laplacian energy, Linear Algebra Appl., 486 (2015), 454-468.

[14] B. Zhou, On Laplacian eigenvalues of a graph, Z. Naturforsch, 59a (2004), 181-184.

Shariefuddin Pirzada Department of Mathematics, University of Kashmir, Srinagar, Kashmir, India

E-mail: pirzadasd@kashmiruniversity.ac.in

Saleem Khan Department of Mathematics, University of Kashmir, Srinagar, Kashmir, India E-mail: khansaleem1727@gmail.com 